Check for updates

Cite this: RSC Adv., 2017, 7, 46354

Received 17th August 2017

Accepted 22nd September 2017

DOI: 10.1039/c7ra09122b

rsc.li/rsc-advances

\section{Adjustment of the solid fluorescence of a chalcone derivative through controlling steric hindrance $\uparrow$}

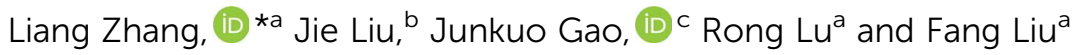

We designed and synthesized a chalcone derivative, ANPEO, which exhibits weak emission in the solution state but high fluorescence efficiency in the solid state $(66 \%)$ because ANPEO shows a coplanar conformation with a dihedral angle $1.58^{\circ}$ and a loose pattern with a mean interlayer distance of approximately $4.21 \AA$.
Many well-known traditional luminogenic materials, such as, polyaromatic hydrocarbons, dicyanomethylene- $4 \mathrm{H}$-pyran, and coumarin derivatives, often possess strong emissions in their solution states; however, their luminescence emissions are always quenched in solutions with high concentrations and in the solid state. ${ }^{1}$ The main cause for quenching is the intense intermolecular $\pi-\pi$ stacking interaction between the aromatic rings of adjoining luminophores, thereby leading to decay or relaxation back to the ground state from the excited state of the luminophore via non-radiative channels. Quenching limits practical applications, thus, researchers have made numerous efforts to design and synthesize new luminogenic materials that show low fluorescence efficiencies in the solution state but their fluorescence efficiencies increase with the increase of concentration and they can emit strong fluorescence in their solid states. ${ }^{2-10}$ Such luminogenic materials are focused on propellerlike luminogens, such as hexaphenylsilole and tetraphenylethenes. Recently, Tang et al. have designed and synthesized a coumarin derivative $(9,10,11,12,13,14$-hexahydro- $6 H$-5-oxa7a,10a-diazacyclohepta[no]tetraphene-6,7(8H)-dione) that exhibits high fluorescence efficiency in its solid state due to its intrinsic structural flexibility and nonplanarity. ${ }^{11}$ Motivated by their successful case, we modified traditional luminogenic materials to achieve strong emissions in the solid state.

Chalcone and its derivatives have displayed numerous applications in biological chemistry activities. ${ }^{12}$ As a member of the $\pi$-conjugated system, the optical properties of chalcone and its derivatives, including nonlinear optical and fluorescence property, have received considerable attention owing to the

\footnotetext{
${ }^{a}$ School of Material Science and Engineering, Yancheng Institute of Technology, Yancheng 224051, Jiangsu, China. E-mail: zhangliang@ycit.edu.cn

${ }^{b}$ State Key Laboratory of Crystal Materials, Shandong University, Jinan, 250100, P. R. China

${ }^{c}$ College of Materials and Textiles, Zhejiang Sci-Tech University, Hangzhou, 310018, China

$\dagger$ Electronic supplementary information (ESI) available. CCDC 1564717 and 1564718. For ESI and crystallographic data in CIF or other electronic format see DOI: $10.1039 / \mathrm{c} 7 \mathrm{ra09122b}$
}

delocalization of electronic charge distribution and overlapping $\pi$ orbitals. ${ }^{13}$ Furthermore, as a family of traditional luminogenic materials, chalcone and its derivatives often possess strong emissions in the solution state but their luminescence are always quenched in high-concentration solutions or/and solid states..$^{14}$ However, their optical properties are easily controlled by introducing electron donor or acceptor groups to aromatic rings. In view of the considerable importance and adjustable structure of chalcone and its derivatives, designing and synthesizing a chalcone that can emit strong fluorescence in solid state is possible.

Herein, we successfully designed and synthesized two chalcone derivatives, 3-(2-(allyloxy)naphthalen-1-yl)-1-phenylprop-2en-1-one (abbreviated as ANPEO) and 1-phenyl-3-(2-((4vinylbenzyl)oxy)naphthalen-1-yl)prop-2-en-1-one (abbreviated as VBNPEO), with similar structures and different steric hindrances (allyl for ANPEO and 4-vinylbenzyl for VBNPEO). ANPEO exhibits weak emission in its solution state but high fluorescence efficiency in its solid state. Moreover, VBNPEO exhibits weak fluorescence in both solution and solid states. Single-crystal X-ray diffraction (SCXRD) data of ANPEO and VBNPEO (from chloroform/ethanol) were collected and analyzed to further understand the relationships between the fluorescence properties and molecular structures of ANPEO and VBNPEO.

The synthetic procedure for preparing ANPEO and VBNPEO is displayed in Scheme 1. Considering the influence of steric hindrance, we synthesized two new intermediate compounds, 2-(allyloxy)-1-naphthaldehyde (abbreviated as AN) and 2-((4vinylbenzyl)oxy)-1-naphthaldehyde (abbreviated as VBN), with different steric hindrances by introducing allyl and 4-vinylbenzyl into 2-hydroxy-1-naphthaldehyde. ANPEO and VBNPEO were further synthesized through an aldol condensation reaction between AN/VBN and acetophenone. ANPEO and VBNPEO were fully characterized and confirmed by ${ }^{1} \mathrm{H}$ and ${ }^{13} \mathrm{C}$ NMR spectroscopy, mass spectrometry, and SCXRD.

The absorption spectra of ANPEO and VBNPEO in different polar solvents are shown in Fig. 1 and S7, $\dagger$ respectively. As exhibited in Fig. 1, the absorption spectrum of ANPEO in 


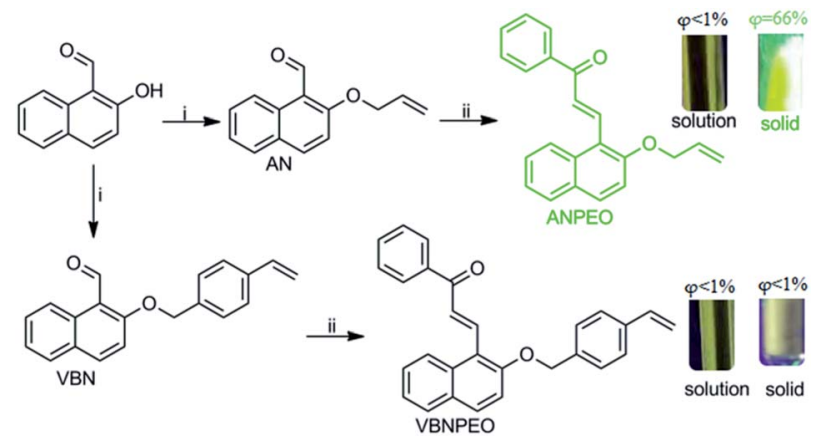

Scheme 1 Synthesis of ANPEO and VBNPEO. (i) potassium carbonate, allyl bromide or 4-vinylbenzyl chloride; (ii) $98 \%$ concentrated sulfuric acid, acetic acid, acetophenone.

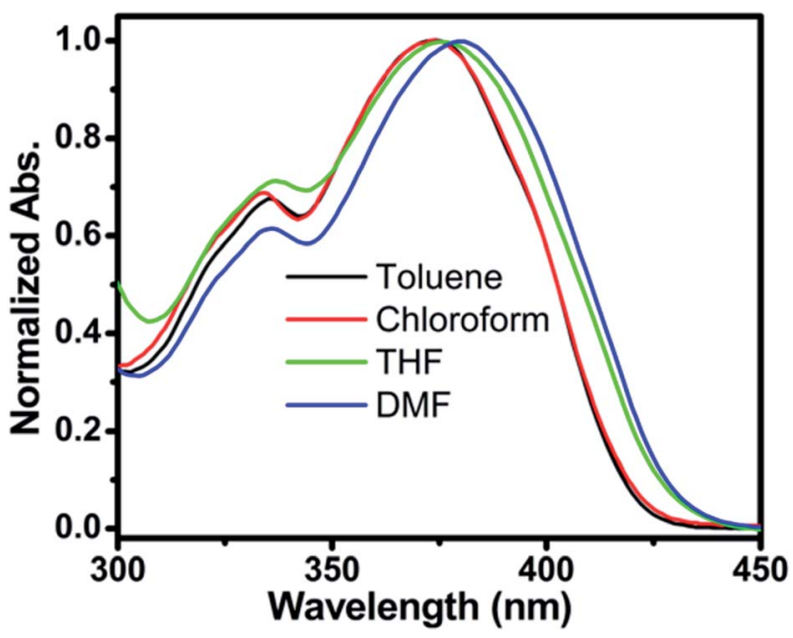

Fig. 1 UV-vis absorption spectra of ANPEO.

toluene (a weak polar solvent) shows two prominent bands at $\lambda_{\max } 335$ and $374 \mathrm{~nm}$, which can be ascribed to a localized aromatic $\pi-\pi^{*}$ transition and charge transfer, respectively. When the polarity of the solvent increased, a slight charge transfer was observed. The absorption wavelength at $\lambda_{\max }$ $374 \mathrm{~nm}$ was red-shifted to $375 \mathrm{~nm}$ to $376 \mathrm{~nm}$ to $380 \mathrm{~nm}$ when the solvent changed from toluene to chloroform to tetrahydrofuran (THF) to $N, N$-dimethylformamide (DMF). The absorption behavior of VBNPEO was similar to that of ANPEO. Compared with the absorption spectrum of ANPEO in toluene solution, the absorption spectrum of VBNPEO in toluene solution was only red-shifted by $\sim 2 \mathrm{~nm}$. Moreover, the emission spectra of ANPEO and VBNPEO were investigated in different solvents. Unlike other chalcone derivatives, ${ }^{14}$ ANPEO and VBNPEO exhibited almost no fluorescence in common solvents, such as toluene and chloroform (Fig. S8 and S9†). To confirm these phenomenon, the fluorescence quantum yields of ANPEO and VBNPEO were also determined in different solvents using quinine bisulfate ( $54.6 \%$ in $0.1 \mathrm{~N} \mathrm{H}_{2} \mathrm{SO}_{4}$ ) as a standard. Subsquently, the fluorescence quantum yields of ANPEO and VBNPEO were much less than $1 \%$ in different solvents. The emission spectra of ANPEO and VBNPEO in the aggregation state were studied in
THF/water mixture to investigate this phenomenon. When water $\left(f_{\mathrm{w}}=10 \%\right)$ was added to the ANPEO THF solution (Fig. 2), the emission wavelength was blue-shifted from $520 \mathrm{~nm}$ to $488 \mathrm{~nm}$ and photoluminescence (PL) intensity increased. However, the emission wavelength was red-shifted from $488 \mathrm{~nm}$ to $518 \mathrm{~nm}$ as the water fraction changed from $20 \%$ to $80 \%$. The PL intensity increased as the water fraction increased from $20 \%$ to $60 \%$, but slightly decreased when the water fraction further increased from $60 \%$ to $80 \%$. When the water fraction increased to $90 \%$, the emission wavelength was blue-shifted to $470 \mathrm{~nm}$ and PL intensity further increased. The different behaviors of ANPEO in THF/water mixture solution was caused by the different aggregation states of ANPEO in the THF/water mixture solution, as confirmed by scanning electron microscopy. As shown in Fig. S10, $\dagger$ the aggregating states of ANPEO in THF/ water mixture solution from $20 \%$ to $40 \%$, from $50 \%$ to $60 \%$, and from $70 \%$ to $80 \%$ are similar. The different aggregating states suggest that ANPEO exhibits different morphologies, leading to different fluorescence behaviors. The emission behavior of VBNPEO in THF/water mixture was similar to that of ANPEO (Fig. S11 and S12†). We further investigated the emission spectra of ANPEO and VBNPEO in the solid state. As displayed in Fig. 3, solid ANPEO emits a strong green light at $\lambda_{\max }$ $514 \mathrm{~nm}$ with its fluorescence quantum yield as high as $66 \%$. However, solid VBNPEO exhibited almost no fluorescence (Fig. S13†).

SCXRD data of ANPEO and VBNPEO (from chloroform/ ethanol, CCDC: 1564717 for ANPEO and 1564718 for VBNPEO $\dagger$ ) were collected and analyzed to further understand the relationships between molecular structures and fluorescence properties of ANPEO and VBNPEO. To demonstrate the coplanarity of the molecules, two benzene rings (named as $\alpha$ and $\beta$, Fig. 4) were chosen as the basic planes and the

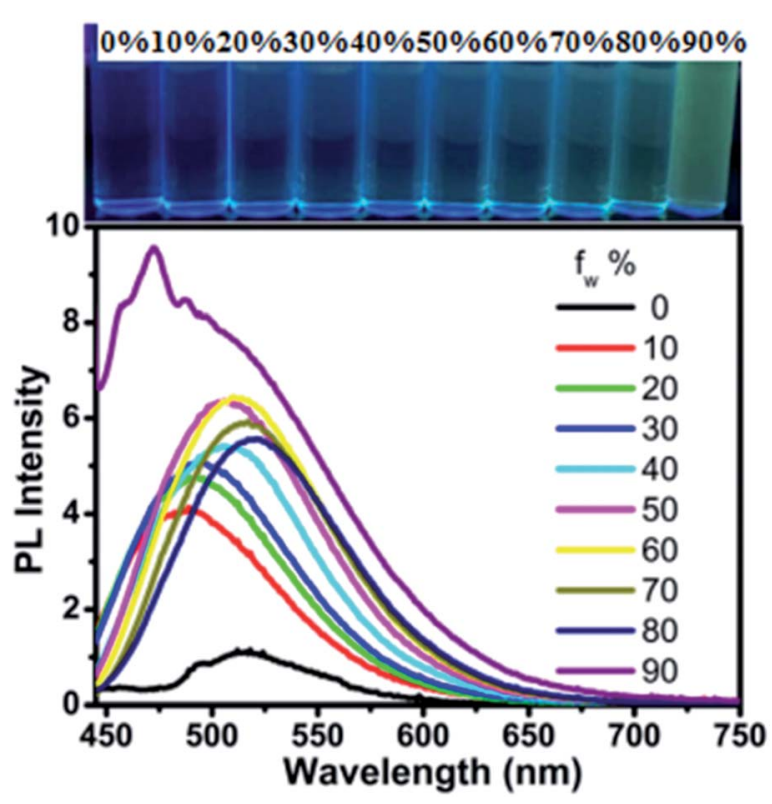

Fig. 2 Emission spectra of ANPEO in the THF/water mixtures $(4 \times$ $10^{-4} \mathrm{~mol} \mathrm{~L}^{-1}$ ). Photographs were taken under $365 \mathrm{~nm}$ UV light. 


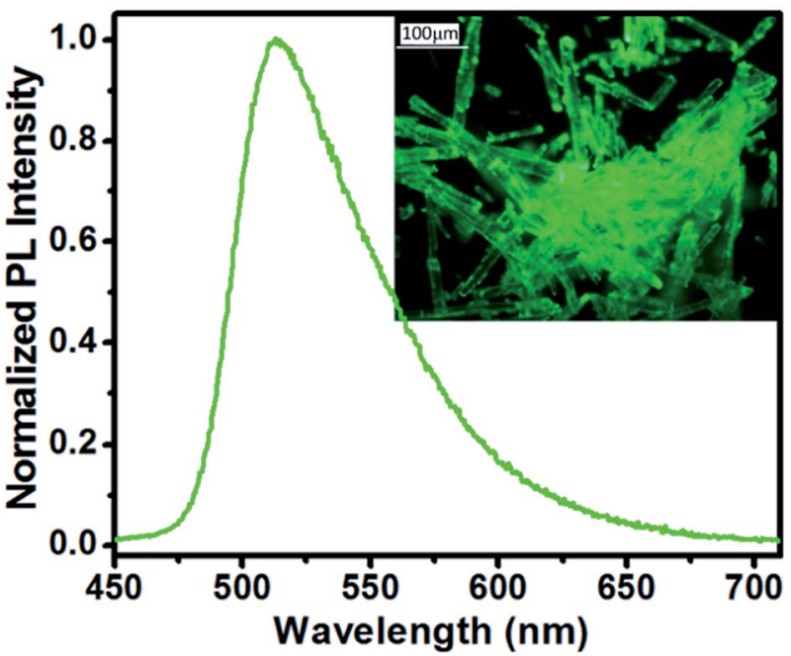

Fig. 3 Emission spectrum of solid ANPEO (inset: excitation wavelength 325-365 nm, exposure time $200 \mathrm{~ms}$ ).

corresponding dihedral angles between the two planes were $1.58^{\circ}$ for ANPEO and $32.33^{\circ}$ for VBNPEO, suggesting that ANPEO exhibits better coplanarity than VBNPEO. Generally, molecules with high coplanar conformation will benefit for fluorescence efficiency because of the bigger $\pi$-electron delocalization. ${ }^{15}$ Subsequently, allyloxy group (low steric hindrance) is little effect on ANPEO which shows a planar conformation, while vinylbenzyl group (high steric hindrance) causes VBNPEO to exhibit a distorted conformation rather than a planar conformation. The weak fluorescence of ANPEO and VBNPEO in solution may be due to two reasons: (1) ANPEO and VBNPEO exhibit low planarity with small $\pi$-electron delocalization; (2) the motion of a chemical bond is strong, which leads to decay or relaxation back to the ground state from the excited state of the luminophore via non-radiative channels. Furthermore, although ANPEO showed a coplanar conformation in solid state, the molecules were arranged in a loose pattern with a mean interlayer distance of approximately $4.21 \AA$, indicating no $\pi-\pi$ intermolecular interactions between the $\pi$-plane of the neighboring molecules, leading to the high-fluorescence quantum yield of ANPEO. The molecules of VBNPEO arranged in a more loose pattern with a mean interlayer distance of approximately $4.81 \AA$, due to high steric hindrance of vinylbenzyl group.

To gain a clear understanding of the relationships between molecular structure, electronic structure, and fluorescence property of ANPEO and VBNPEO. The ground-state geometry of ANPEO and VBNPEO were optimized via the hybrid density functional theory (B3LYP) with 6-31G* basis set using the Gaussian 03 program package. As shown in Fig. S14a and b, $\dagger$ the highest occupied molecular orbital (HOMO) of ANPEO is a $\pi$ orbital and the electron density is spread to chalcone and naphthalene groups, while the lowest unoccupied molecular orbital (LUMO) is of $\pi^{*}$ character and is distributed on the whole molecule except allyloxy group. Thus, there is no obvious change in the electron density of allyloxy group. The electron density of VBNPEO is similar to that of ANPEO. The electron density of vinylbenzyl group is also no obvious change, suggesting that allyloxy and vinylbenzyl group exhibit little effect on the electronic structure. As shown in Fig. S14c and $f, \uparrow$ the coplanarity of ANPEO and VBNPEO are poor at ground-state with dihedral angles of $87.17^{\circ}$ and $39.66^{\circ}$, respectively. The low planarity indicates a small $\pi$-electron delocalization, leading to a weak fluorescence of ANPEO and VBNPEO in (a)

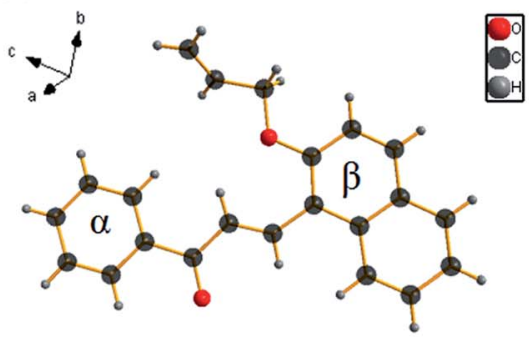

(b)

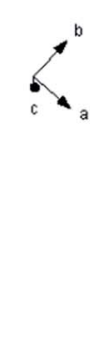

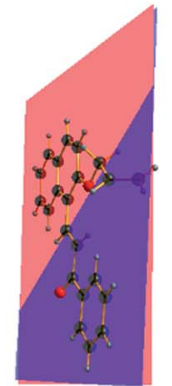
$L_{(\alpha, \beta)}=1.58^{\circ}$ (c)

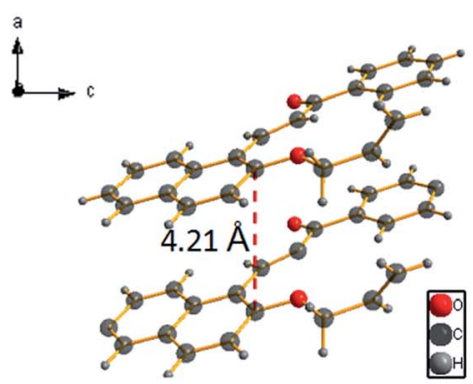

(d)

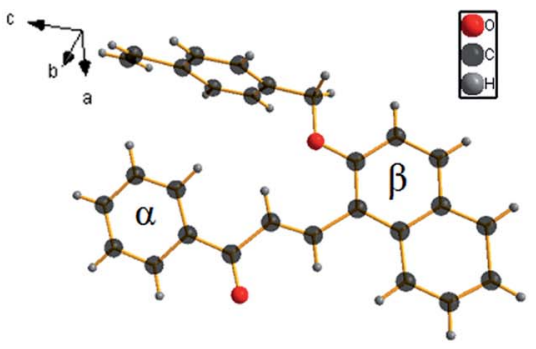

(e)

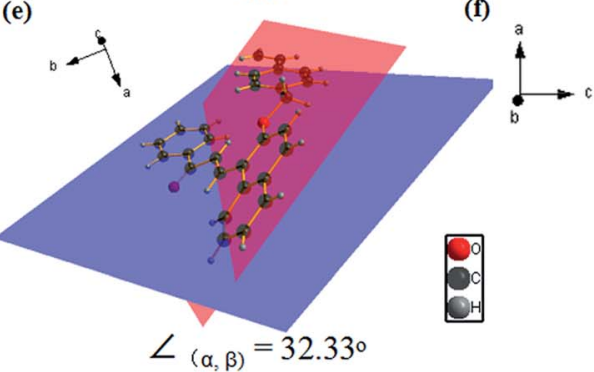

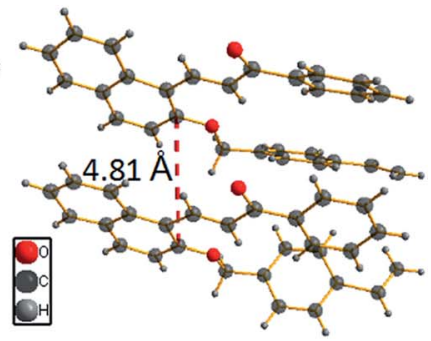

Fig. 4 Crystal structures, the related dihedral angles, and the molecular stacking patterns and relative mean distances of ANPEO (a-c) and $\operatorname{VBNPEO}(d-f)$ interlayers. 
solution. However, ANPEO exhibits high planarity at solid state from Fig. 4, while VBNPEO still displays poor planarity at solid state from Fig. 4, leading to a high fluorescence of ANPEO at solid state.

We successfully designed and synthesized two chalcone derivatives, ANPEO and VBNPEO, with similar structures and different steric hindrances (allyl for ANPEO and 4-vinylbenzyl for VBNPEO). ANPEO exhibits weak emission in the solution state but high fluorescence efficiency in the solid state (66\%). Moreover, VBNPEO shows weak fluorescence in both solution and solid states. On the basis of single-crystal structural analysis, ANPEO exhibits a coplanar conformation with a dihedral angle as low as $1.58^{\circ}$, which is different from that of VBNPEO, with a dihedral angle as high as $32.33^{\circ}$. Furthermore, the ANPEO molecules are arranged in a loose pattern with a mean interlayer distance of approximately $4.21 \AA$, indicating no $\pi-\pi$ intermolecular interaction between the $\pi$-plane of the neighboring molecules. Our results strongly suggest that the solid fluorescence of chalcone derivatives can be tunable by controlling steric hindrance.

\section{Conflicts of interest}

There are no conflicts to declare.

\section{Notes and references}

1 (a) Q. Xu, C. H. Heo, G. Kim, H. W. Lee, H. M. Kim and J. Yoon, Angew. Chem., Int. Ed., 2015, 54, 4890; (b) Z. Guo, W. Zhu and H. Tian, Chem. Commun., 2012, 48, 6073; (c) J. J. Shie, Y. C. Liu, Y. M. Lee, C. Lim, J. M. Fang and C. H. Wong, J. Am. Chem. Soc., 2014, 136, 9953; (d) Q. Zhang, H. Kuwabara, W. J. Potscavage Jr, S. Huang, Y. Hatae, T. Shibata and C. Adachi, J. Am. Chem. Soc., 2014, 136, 18070; (e) P.-Y. Gu, J. Gao, Q. Zhang, G. Liu, F. Zhou, Q.-F. Xu and J.-M. Lu, J. Mater. Chem. C, 2014, 2, 1539; $(f)$ J. Xiao, Y. Divayana, Q. Zhang, H. M. Doung, H. Zhang, F. Boey, X. W. Sun and F. Wudl, J. Mater. Chem., 2010, 20, 8167.

2 (a) Z. Xie, C. Chen, S. Xu, J. Li, Y. Zhang, S. Liu, J. Xu and Z. Chi, Angew. Chem., Int. Ed., 2015, 54, 7181; (b) T. Yu, D. Ou, Z. Yang, Q. Huang, Z. Mao, J. Chen, Y. Zhang, S. Liu, J. Xu, M. R. Bryce and Z. Chi, Chem. Sci., 2017, 8, 1163.

3 (a) J. Yang, L. Li, Y. Yu, Z. Ren, Q. Peng, S. Ye, Q. Li and Z. Li, Mater. Chem. Front., 2017, 1, 91; (b) C. Wang, B. Xu, M. Li, Z. Chi, Y. Xie, Q. Li and Z. Li, Mater. Horiz., 2016, 3, 220.

4 (a) S. Sekiguchi, K. Kondo, Y. Sei, M. Akita and M. Yoshizawa, Angew. Chem., Int. Ed., 2016, 55, 6906; (b) M. Yamashina, M. M. Sartin, Y. Sei, M. Akita, S. Takeuchi, T. Tahara and M. Yoshizawa, J. Am. Chem. Soc., 2015, 137, 9266.

5 (a) B. Xu, J. He, Y. Dong, F. Chen, W. Yu and W. Tian, Chem. Commun., 2011, 47, 6602; (b) J. Zhang, J. Chen, B. Xu, L. Wang, S. Ma, Y. Dong, B. Li, L. Ye and W. Tian, Chem. Commun., 2013, 49, 3878.
6 (a) H. Naito, Y. Morisaki and Y. Chujo, Angew. Chem., Int. Ed., 2015, 54, 5084; (b) H. Naito, K. Nishino, Y. Morisaki, K. Tanaka and Y. Chujo, Angew. Chem., Int. Ed., 2017, 56, 254. 7 (a) Z. Mao, Z. Yang, Y. Mu, Y. Zhang, Y. F. Wang, Z. Chi, C. C. Lo, S. Liu, A. Lien and J. Xu, Angew. Chem., Int. Ed., 2015, 54, 6270; (b) Z. Chi, X. Zhang, B. Xu, X. Zhou, C. Ma, Y. Zhang, S. Liu and J. Xu, Chem. Soc. Rev., 2012, 41, 3878.

8 (a) R. Li, S. Xiao, Y. Li, Q. Lin, R. Zhang, J. Zhao, C. Yang, K. Zou, D. Li and T. Yi, Chem. Sci., 2014, 5, 3922; (b) C.-M. Chou, S. Nobusue, S. Saito, D. Inoue, D. Hashizume and S. Yamaguchi, Chem. Sci., 2015, 6, 2354; (c) A. Shao, Y. Xie, S. Zhu, Z. Guo, S. Zhu, J. Guo, P. Shi, T. D. James, H. Tian and W. H. Zhu, Angew. Chem., Int. Ed., 2015, 54, 7275; (d) H. Lu, Y. Zheng, X. Zhao, L. Wang, S. Ma, X. Han, B. Xu, W. Tian and H. Gao, Angew. Chem., Int. Ed., 2016, $55,155$.

9 J. Seo, J. W. Chung, J. E. Kwon and S. Y. Park, Chem. Sci., 2014, 5, 4845.

10 (a) Y. Q. Dong, J. W. Lam and B. Z. Tang, J. Phys. Chem. Lett., 2015, 6, 3429; (b) J. Qian, Z. Zhu, A. Qin, W. Qin, L. Chu, F. Cai, H. Zhang, Q. Wu, R. Hu, B. Z. Tang and S. He, Adv. Mater., 2015, 27, 2332.

11 F. Bu, R. Duan, Y. Xie, Y. Yi, Q. Peng, R. Hu, A. Qin, Z. Zhao and B. Z. Tang, Angew. Chem., Int. Ed., 2015, 54, 14492.

12 (a) S. Satbhaiya and O. P. Chourasia, RSC Adv., 2015, 5, 84810; (b) W. Lin, L. Yuan, Z. Cao, Y. Feng and L. Long, Chem.-Eur. J., 2009, 15, 5096; (c) Z. Nowakowska, Eur. J. Med. Chem., 2007, 42, 125; (d) G.-J. Kim, K. Lee, H. Kwon and H.-J. Kim, Org. Lett., 2011, 13, 2799.

13 (a) E. D. D'Silva, D. Narayan Rao, R. Philip, R. J. Butcher, Rajnikant and S. M. Dharmaprakash, J. Phys. Chem. Solids, 2011, 72, 824; (b) T. Chandra Shekhara Shetty, S. Raghavendra, C. S. Chidan Kumar and S. M. Dharmaprakash, Opt. Laser Technol., 2016, 77, 23; (c) P. S. Patil, S. R. Maidur, S. V. Rao and S. M. Dharmaprakash, Opt. Laser Technol., 2016, 81, 70; (d) C. Shekhara Shetty, S. Raghavendra, C. S. Chidan Kumar and S. M. Dharmaprakash, Appl. Phys. B, 2016, 122; (e) L. M. G. Abegão, R. D. Fonseca, F. A. Santos, G. B. Souza, A. L. B. S. Barreiros, M. L. Barreiros, M. A. R. C. Alencar, C. R. Mendonça, D. L. Silva, L. De Boni and J. J. Rodrigues, Chem. Phys. Lett., 2016, 648, 91.

14 (a) E. Yang, C. Ruzie, M. Krayer, J. R. Diers, D. M. Niedzwiedzki, C. Kirmaier, J. S. Lindsey, D. F. Bocian and D. Holten, Photochem. Photobiol., 2013, 89, 586; (b) Q. Zhou, P. Gu, Y. Zhang, N. Li, Q. Xu, Y. Zhang and J. Lu, Chin. J. Chem., 2014, 32, 573; (c) K. G. Komarova, S. N. Sakipov, V. G. Plotnikov and M. V. Alfimov, J. Lumin., 2015, 164, 57.

15 (a) Y. Cheng, Y. Qi, Y. Tang, C. Zheng, Y. Wan, W. Huang and R. Chen, J. Phys. Chem. Lett., 2016, 7, 3609; (b) T. J. Fauvell, T. Zheng, N. E. Jackson, M. A. Ratner, L. Yu and L. X. Chen, Chem. Mater., 2016, 28, 2814. 